\title{
Optimizing the regimes of the Advanced LIGO gravitational wave detector for multiple source types
}

\author{
I. S. Kondrashov, ${ }^{1}$ D. A. Simakov, ${ }^{1}$ F. Ya. Khalili, ${ }^{1, *}$ and S. L. Danilishin ${ }^{2,1,+}$ \\ ${ }^{1}$ Physics Faculty, Moscow State University, Moscow 119992, Russia \\ ${ }^{2}$ Max-Planck-Institut für Gravitationsphysik (Albert-Einstein-Institut), Golm D-14482, Germany \\ (Received 16 June 2008; published 18 September 2008)
}

\begin{abstract}
We developed algorithms which allow us to find regimes of the signal-recycled Fabry-Perot-Michelson interferometer [for example, the Advanced Laser Interferometric Gravitational Wave Observatory (LIGO)], optimized concurrently for two (binary inspirals + bursts) and three (binary inspirals + bursts + millisecond pulsars) types of gravitational wave sources. We show that there exists a relatively large area in the interferometer parameters space where the detector sensitivity to the first two kinds of sources differs only by a few percent from the maximal ones for each kind of source. In particular, there exists a specific regime where this difference is $\approx 0.5 \%$ for both of them. Furthermore, we show that even more multipurpose regimes are also possible that provide significant sensitivity gain for millisecond pulsars with only minor sensitivity degradation for binary inspirals and bursts.
\end{abstract}

DOI: 10.1103/PhysRevD.78.062004

PACS numbers: 04.80.Nn, 07.60.Ly, 95.55.Ym

\section{INTRODUCTION}

Within the last decade we have witnessed very significant progress in experimental gravitational wave (GW) astronomy. All the ground-based interferometric GW antennas such as LIGO [1] in the USA, VIRGO [2] in Italy, GEO600 [3] in Germany, and TAMA300 [4] in Japan have been commissioned to operation and have started to record scientific data. Nevertheless, no signs of gravitational waves were found thus far in these data which is, as we understand now, quite reasonable as it agrees with moderately optimistic predictions of the astrophysicists on the rate of measurable events within the limits of the antennas detection range. This possibility was realized by the GW community, and work on the design of the next, second generation of GW antennas went on in parallel with efforts in enhancement of the first generation ones. A pioneer among the second generation GW detectors will become an American Advanced LIGO project whose construction should start in 2010 [5]. It is planned to have sensitivity more than an order of magnitude higher than its predecessor. Such a dramatic increase will be provided by a significantly lower seismic noise level due to new active antiseismic isolation, the use of higher quality optics, and a lower level of quantum noise.

The main difference between the initial LIGO and the Advanced LIGO designs that is crucial for lowering this noise is the use of the signal recycling (SR) technique first suggested by Meers in his pioneering work [6]. Its implementation in contemporary detector setup is relatively easy as it requires one to install only one additional mirror in the

\footnotetext{
*farid@hbar.phys.msu.ru

${ }^{+}$Stefan.Danilishin@aei.mpg.de
}

interferometer output port. This mirror reflects the sideband signal field coming out of the interferometer back to the arm cavities or "recycles" it. However, the dynamics and quantum noise properties of the interferometer become much richer and thus provide more freedom in adjusting its sensitivity curve to fit the current research goals. In particular, it was stressed by Buonnano and Chen [7] that the optical system composed of the SR cavity and the arm cavities forms a composite resonant cavity whose eigenfrequencies and quality factors can be controlled by the position and reflectivity of the SR mirror, thus increasing or decreasing the storage time of the signal inside the cavity. Moreover, as in Advanced LIGO it is planned to increase the optical power, circulating in the arm cavities approximately 80 times with respect to the initial LIGO, an optomechanical interaction between laser field and mirrors will significantly influence the dynamics of the test masses turning them from free bodies (within the detection frequency band, $\sim 10-10^{4} \mathrm{~Hz}$ ) into oscillators with eigenfrequency falling into the detection band. This effect, known as ponderomotive rigidity [7-11], arises when an offresonant optical field creates an effective restoring force originating from radiation pressure, which occurs as a function of mirror displacement. In this situation one can say that the optical field creates a frequency-dependent mechanical rigidity $[7,10,12-15]$.

As a result, the quantum noise spectral density of signalrecycled interferometers (SRI) can be tuned to provide the best sensitivity for different gravitational wave sources. So far, it was supposed that in order to reach good sensitivity for each of the source types, totally different strategies should be used, which correspond to different optical parameters sets. Detection of gravitational waves from inspiraling neutron star (NS) binaries requires, for ex- 
ample, that the noise spectral density be as small as possible at low and medium frequencies, $f \lesssim 100 \mathrm{~Hz}$. If one is interested in narrow-band detection of GWs from the source with well-defined center frequency $f_{\text {puls }}$, such as high-frequency pulsars, the optimal regime of the interferometer will be absolutely different: evidently, one should choose such a set of optical parameters that provides minimum to noise at $f_{\text {puls. }}$. Searches of GWs from supernovae bursts or stochastic relic gravitational radiation, where analytical waveforms are not known, require flat broadband noise curves [16-18].

Extensive studies of optimal modes of operation of the Advanced LIGO interferometer most suitable for different specific kinds of sources of GWs were carried out in the AdvLIGO Lab in extensive detail [19]. However, in this paper we focus on finding multipurpose regimes of signalrecycled interferometers which might provide good (suboptimal) sensitivity simultaneously for different gravitational wave source types. The possibility to do this originates from the fact that the classical noise budget for currently operating and future GW detectors masks low and medium frequency features of the quantum noise (see Fig. 5) that are mostly susceptible to the variation of optical parameters of the interferometer. This fact, although being quite unpleasant for the tunability of a GW detector for specific sources is surprisingly advantageous for tuning the antenna to have high enough sensitivity to GWs from various types of astrophysical sources simultaneously. As will be shown in subsequent sections the sensitivity changes relatively slowly within quite a wide range of main SRI optical parameters for different types of signals (GWs from inspiraling compact binaries, GW bursts, highfrequency pulsars, etc.). And the fact that these areas for different sources significantly overlap allows one to find quasioptimal regime for two or even three different $\mathrm{GW}$ sources simultaneously. It is shown by the example of Advanced LIGO SRI that rather significant improvement in sensitivity to GW bursts and GWs from high-frequency sources is possible at the cost of quite moderate deterioration of signal strength for compact binary systems. This is illustrated in Figs. 1 and 2 where the relative improvement in signal-to-noise-ratio (SNR) for GW bursts and highfrequency pulsars, correspondingly, is plotted with respect to relative deterioration of SNR for NS, provided that one diverts optical parameters of SRI from the optimal ones for NS. It should also be emphasized here that in spite of Advanced LIGO being used as an example for which we perform calculations, the results we obtain are general and applicable to all SRI limited by classical noise at low and medium frequencies. It is also instructive to mention that our optimization includes only the most basic parameters and the results can be considered only as preliminary guidelines for designing a future generation of GW interferometers. For more specific optimization of the real device a much larger parameter space should be consid-

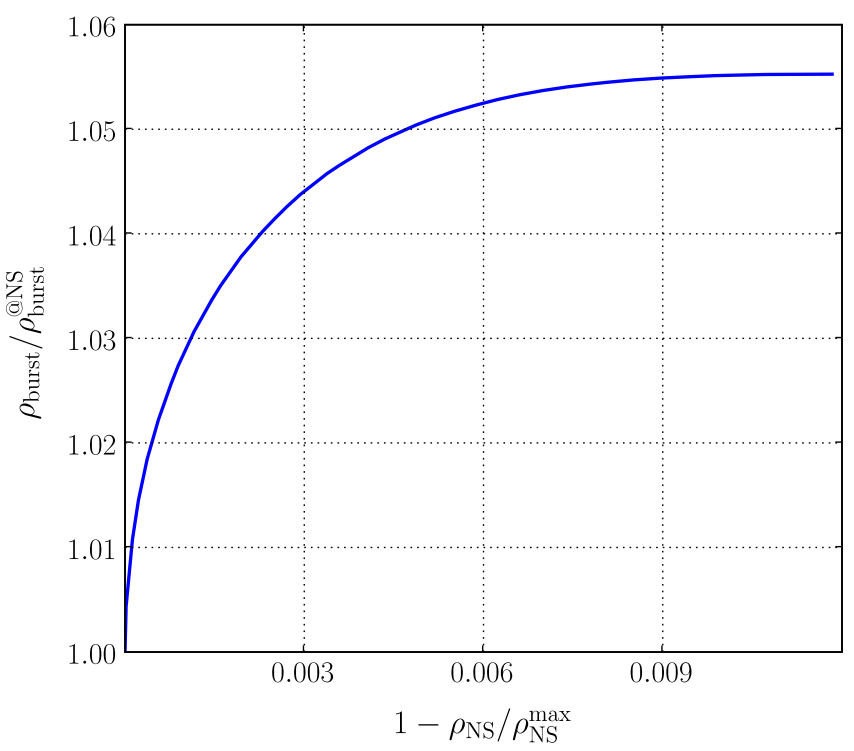

FIG. 1 (color online). Relative improvement in signal-to-noise ratio $\rho_{\text {burst }} / \rho_{\text {burst }}^{@ N S}$ for bursts of GW radiation as a function of relative deterioration in SNR for NS binaries $1-\rho_{\mathrm{NS}} / \rho_{\mathrm{NS}}^{\max }$. Here $\rho_{\text {burst }}^{@ N S}$ is the value of SNR for GW burst sources when the interferometer is tuned for reaching maximum SNR for NS binaries.

ered. Moreover, two different groups of parameters should be treated separately, namely, those that can be changed with relative ease when the device is already working, and those that should be fixed at the design stage and cannot be

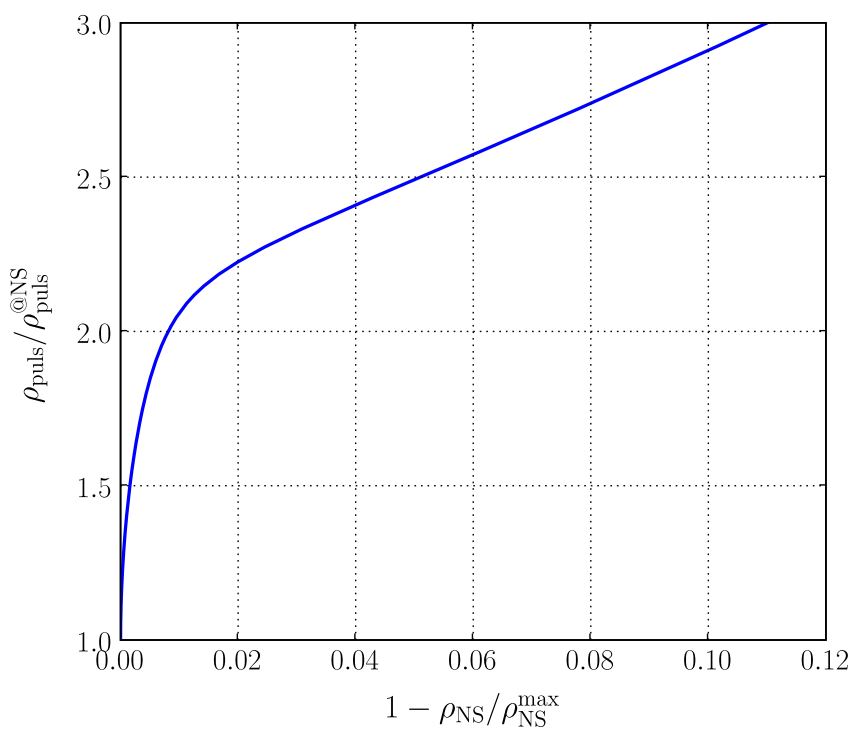

FIG. 2 (color online). Relative improvement in signal-to-noise ratio $\rho_{\text {puls }} / \rho_{\text {puls }}^{@ N S}$ for one of the high-frequency pulsars (J00340534 with rotational frequency $f_{0} \simeq 532,7 \mathrm{~Hz}$ ) as a function of relative deterioration in SNR for NS binaries $1-\rho_{\mathrm{NS}} / \rho_{\mathrm{NS}}^{\max }$. Here $\rho_{\text {puls }}^{@ N S}$ is the value of SNR for GWs from specified pulsar when the interferometer is tuned for reaching maximum SNR for NS binaries. 
TABLE I. Notations used for characterizing the quantum noise of SRI.

\begin{tabular}{lc}
\hline \hline Value & Description \\
\hline$M$ & Test bodies reduced mass \\
$c$ & Speed of light \\
$L$ & SRI arms length \\
$\omega_{p}$ & Laser frequency \\
$\Omega$ & Mechanical frequency \\
$W$ & Circulating optical power \\
$\gamma$ & Effective SRI half-bandwidth \\
$\delta$ & Effective SRI detuning \\
$J=\frac{4 \omega_{p} W}{M c L}$ & Renormalized optical power \\
$\phi$ & Homodyne angle \\
$\eta$ & Total readout quantum efficiency (incl. losses) \\
\hline \hline
\end{tabular}

tuned afterward. The above issues will be considered in future works.

The paper is organized as follows. In Sec. II a brief consideration of quantum noise of the signal-recycled Fabry-Pérot-Michelson interferometer is performed. In Sec. III expressions for the signal-to-noise ratio and the detection range for the gravitational wave radiation from the inspiraling binary system are given and the numerical optimization procedure with respect to interferometer optical parameters is described. The quantitative and qualitative analysis of the obtained results of optimization against GWs from neutron star binaries is performed. In Sec. IV the sensitivity of the interferometer to GW bursts is analyzed and regimes for simultaneous detection of these two types of sources are investigated. In Sec. V this analysis is extended also to high-frequency quasiperiodic sources (pulsars). In Sec. VI the obtained results are discussed and some concluding remarks are given. Some notations and values of parameters used through this paper are listed in Table I.

\section{QUANTUM NOISE OF SIGNAL-RECYCLED INTERFEROMETERS}

In Fig. 3 the schematic drawing of a signal-recycled interferometer is presented. Here the additional signal recycling mirror (SRM) forms, together with the input test masses (ITMs) of arm cavities, an additional SR cavity whose properties are defined by two parameters of SRM, namely, its amplitude reflectivity $\rho$ and detuning phase $\phi_{\mathrm{SRC}}=\left[\omega_{p} l / c\right]_{\bmod 2 \pi}$ gained by the carrier light traveling one way in the SR cavity ( $l$ is the length of the SR cavity).

As demonstrated by Buonnano and Chen [13], there exists one-to-one transformation ("scaling law") between the parameters of a single detuned Fabry-Perot (FP) cavity with one movable mirror and ones of SRI that allow one to describe the optical behavior of it in terms of a much more simple equivalent system such as the FP cavity. According to the scaling law for any SRI there exists a unique FP cavity with bandwidth $\gamma$ and detuning $\delta$ defined by for-

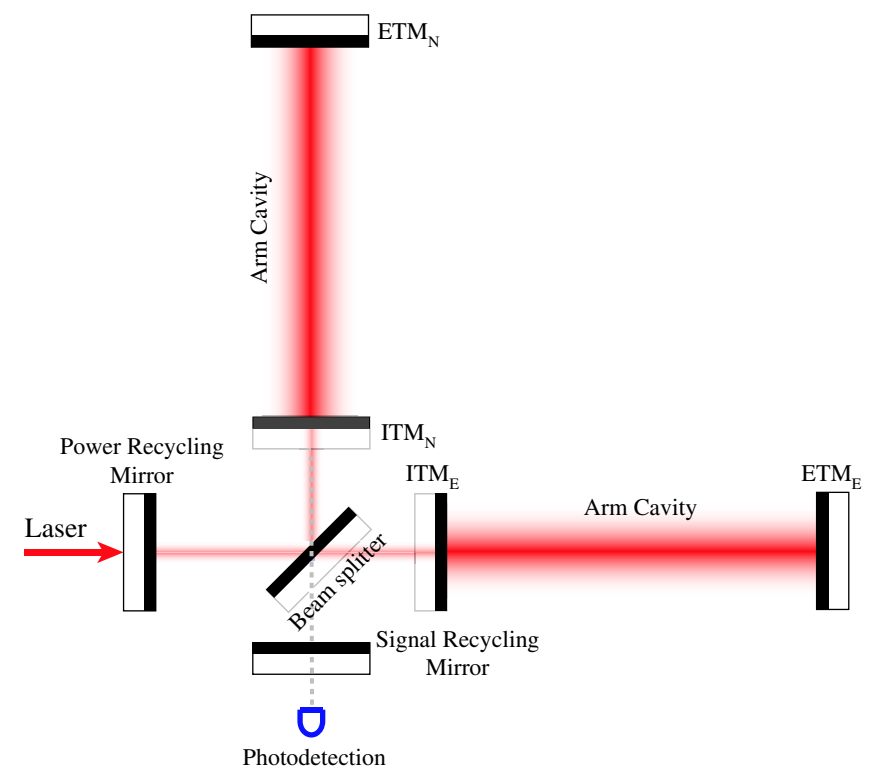

FIG. 3 (color online). Principle optical scheme of signalrecycled interferometer of Advanced LIGO GW detector.

mulas:

$$
\begin{aligned}
& \gamma=\frac{\left(1-\rho^{2}\right) \gamma_{\mathrm{ARM}}}{1+2 \rho \cos 2 \phi_{\mathrm{SRC}}+\rho^{2}}, \\
& \delta=\frac{2 \rho \gamma_{\mathrm{ARM}} \sin 2 \phi_{\mathrm{SRC}}}{1+2 \rho \cos 2 \phi_{\mathrm{SRC}}+\rho^{2}},
\end{aligned}
$$

where $\gamma_{\mathrm{ARM}}=c T / 4 L$ is the half-bandwidth of arm FP cavities that has the same optomechanical features and therefore the same sensitivity as the initial SRI. The effective optical power circulating in the equivalent FP cavity should be twice as large as real optical power $W$ circulating in a single arm cavity. The same is referred to masses of input (ITM) and end (ETM) test masses of effective cavity: $M_{\text {eff }}=2 M$.

Below we will use also extensively the following convenient parameters: generalized bandwidth,

$$
\Gamma=\sqrt{\gamma^{2}+\delta^{2}}=\gamma_{\mathrm{ARM}} \sqrt{\frac{1-2 \rho \cos 2 \phi_{\mathrm{SRC}}+\rho^{2}}{1+2 \rho \cos 2 \phi_{\mathrm{SRC}}+\rho^{2}}}
$$

and detuning phase

$$
\beta=\arctan \frac{\delta}{\gamma}=\arctan \left(\frac{2 \rho}{1-\rho^{2}} \sin 2 \phi_{\mathrm{SRC}}\right)
$$

The above expressions can be also easily reverted to obtain the SR cavity parameters: 


$$
\begin{aligned}
\rho & =\sqrt{\frac{\gamma_{\mathrm{ARM}}^{2}-2 \gamma_{\mathrm{ARM}} \Gamma \cos \beta+\Gamma^{2}}{\gamma_{\mathrm{ARM}}^{2}+2 \gamma_{\mathrm{ARM}} \Gamma \cos \beta+\Gamma^{2}}}, \\
\phi_{\mathrm{SRC}} & =\frac{1}{2} \begin{cases}\arcsin \left(\frac{1-\rho^{2}}{2 \rho} \tan \beta\right), & \Gamma<\gamma_{\mathrm{ARM}}, \\
\pi-\arcsin \left(\frac{1-\rho^{2}}{2 \rho} \tan \beta\right), & \Gamma>\gamma_{\mathrm{ARM}} .\end{cases}
\end{aligned}
$$

Using the scaling law approach, consider the FP cavity with movable mirrors pumped by laser light with frequency $\omega_{0}$. The action of a gravitational wave on such a system can be effectively described by means of effective forces acting on the mirrors and therefore changing dynamically the phase shift of outgoing light with respect to ingoing light.

There exist two kinds of quantum fluctuations limiting the sensitivity of detector. They are the so-called laser shot noise and radiation pressure noise (RPN). The first one originates from quantum fluctuations of the electromagnetic wave phase which prevents one from the exact phase shift measurement and is, in essence, measurement accuracy. The second one, being a consequence of fluctuations of the light amplitude, causes random radiation pressure force to move the mirrors and masks the measured signal force. As far as this noise is the direct consequence of the measurement, it is also known as backaction noise because of the backaction of the measurement device (laser light) on the measured quantity (phase shift).

It is convenient to describe this system in terms of linear quantum measurement theory formalism developed in [20] and thoroughly elaborated for use in gravitational wave interferometers in [7]. Following this formalism we can represent our meter as two linearly coupled systems, probe (test masses), and detector (laser light and photodetectors). The schematic drawing of this equivalent linear system is presented in Fig. 4. Here $\hat{x}$ stands for some measured observable of the probe (mirrors relative displacement in our case), $\hat{F}$ is some observable of the detector through which it is coupled to the probe (radiation pressure force in our case), $G$ is the classical signal force being detected (GW action on the detector), and $\hat{Z}$ is the measured observable of the detector (output light quadrature in our case). Following [13] we write down the Hamiltonian of

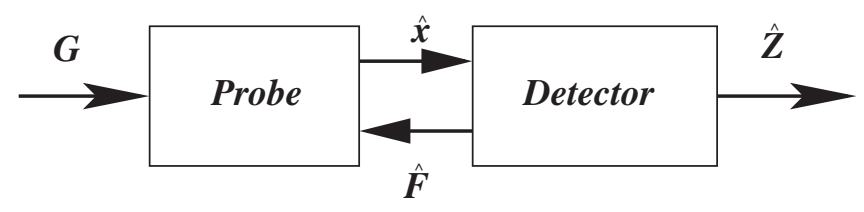

FIG. 4. Schematic diagram of the quantum measurement device. $G$ is the classical observable (e.g.,force) acting on the probe that is measured. $\hat{Z}$ is the output signal of the detector device, $\hat{x}$ is the linear observable (e.g., displacement) of the probe, and $\hat{F}$ is the linear observable of the detector which describes the backaction force on the probe. our system as

$$
\hat{H}=\left[\left(\hat{H}_{\mathcal{P}}-\hat{x} G\right)+\hat{H}_{\mathcal{D}}\right]-\hat{x} \hat{F} \equiv \hat{H}^{(0)}+\hat{V},
$$

where $\hat{H}^{(0)}=\left(\hat{H}_{\mathcal{P}}-\hat{x} G\right)+\hat{H}_{\mathcal{D}}$ is considered as a zeroth order Hamiltonian for both the detector (marked by $\mathcal{D}$ ) and the probe (marked by $\mathcal{P}$ ) subsystems, and linear coupling between them $\hat{V}=-\hat{x} \hat{F}$ considered as a perturbative Hamiltonian. Using this approach, one can write down all of the observables of the system as a sum of unperturbed zeroth order terms [marked by superscript (0)] and perturbations [marked by superscript (1)] [see Eqs. (2.12)-(2.14) of [13]]. In the frequency domain these observables are read as

$$
\begin{aligned}
& \hat{Z}^{(1)}(\Omega)=\hat{Z}^{(0)}(\Omega)+R_{Z F}(\Omega) \hat{x}^{(1)}(\Omega), \\
& \hat{F}^{(1)}(\Omega)=\hat{F}^{(0)}(\Omega)+R_{F F}(\Omega) \hat{x}^{(1)}(\Omega), \\
& \hat{x}^{(1)}(\Omega)=\hat{x}^{(0)}(\Omega)+\operatorname{Lh}(\Omega) / 2+R_{x x}(\Omega) \hat{F}^{(1)}(\Omega) .
\end{aligned}
$$

Here $L h(\Omega) \equiv R_{x x} G(\Omega)$ is the GW signal proportional to metrics variation spectrum $h(\Omega)$. Quantities $R_{A B}(\Omega)$ are frequency-dependent susceptibilities, in particular, $R_{Z F}(\Omega)$ is the optomechanical coupling factor,

$$
R_{x x}(\Omega)=-\frac{1}{M \Omega^{2}}
$$

is the mechanical susceptibility of the SRI, and

$$
R_{F F}(\Omega)=\frac{M J \delta}{\Gamma^{2}-\Omega^{2}-2 i \gamma \Omega}
$$

is the optical rigidity. $\hat{Z}^{(0)}$ corresponds to shot noise of the laser light, $x^{(0)} \equiv x_{\text {tech }}$ stands for any displacement noise sources associated with the test mass reflecting surface with respect to its center of mass position, namely, thermoelastic and Brownian noise of the mirror coatings and substrate, and

$$
\hat{F}^{(0)}(\Omega)=\hat{F}_{\mathrm{RPN}}(\Omega)+F_{\text {tech }}(\Omega),
$$

where $\hat{F}_{\mathrm{RPN}}(\Omega)$ is the radiation pressure noise and $F_{\text {tech }}(\Omega)$ is describing all the classical force noises, most notably suspension thermal, gravity gradient, and seismic noises.

Using these definitions one can now write down the output of the SRI reduced to metrics variation $h$ units as

$$
\begin{aligned}
\hat{h}_{\text {out }}(\Omega)= & h(\Omega)+\frac{2}{L}\left\{R_{x x}(\Omega)\left[\hat{F}_{\mathrm{RPN}}(\Omega)+F_{\text {tech }}(\Omega)\right]\right. \\
& \left.+\left[R_{x x}(\Omega) R_{F F}(\Omega)+1\right]\left[\hat{x}_{\mathrm{SN}}(\Omega)+x_{\text {tech }}(\Omega)\right]\right\},
\end{aligned}
$$

where

$$
\hat{x}_{\mathrm{SN}}(\Omega)=\frac{\hat{Z}^{(0)}}{R_{Z F}(\Omega)}
$$

is the normalized shot noise. 
Accounting for these definitions, one can write down spectral density of the interferometer output noise as

$$
S^{h}(\Omega)=S_{\text {quant }}^{h}(\Omega)+S_{\text {tech }}^{h}(\Omega) .
$$

Here

$$
\begin{aligned}
S_{\text {quant }}^{h}(\Omega)= & \frac{4}{L^{2}}\left(\left|R_{x x}(\Omega) R_{F F}(\Omega)+1\right|^{2} S_{x}^{\mathrm{SN}}(\Omega)\right. \\
& +2 \Re\left\{\left[R_{x x}(\Omega) R_{F F}(\Omega)+1\right]^{*} S_{x F}(\Omega)\right\} \\
& \left.+\left|R_{x x}(\Omega)\right|^{2} S_{F}^{\mathrm{RPN}}(\Omega)\right)
\end{aligned}
$$

is the sum quantum noise spectral density,

$$
S_{x}^{\mathrm{SN}}(\Omega)=\frac{\hbar}{2 M J \gamma \eta} \frac{\Omega^{4}+2 \Gamma^{2} \Omega^{2} \cos 2 \beta+\Gamma^{4}}{\Gamma^{2} \cos ^{2}(\beta+\phi)+\Omega^{2} \cos ^{2} \phi}
$$

is the shot noise (10) spectral density,

$$
S_{F}^{\mathrm{RPN}}(\Omega)=\frac{2 \hbar M J \gamma\left(\Gamma^{2}+\Omega^{2}\right)}{\Omega^{4}+2 \Gamma^{2} \Omega^{2} \cos 2 \beta+\Gamma^{4}}
$$

is the radiation pressure noise $\hat{F}_{\text {RPN }}(\Omega)$ spectral density,

$$
S_{x F}(\Omega)=\hbar \frac{\Gamma \sin (\beta+\phi)+i \Omega \sin \phi}{\Gamma \cos (\beta+\phi)+i \Omega \cos \phi}
$$

is their spectral cross-correlation function,

$$
\begin{aligned}
S_{\text {tech }}^{h}(\Omega)= & \frac{4}{L^{2}}\left(\left|R_{x x}(\Omega) R_{F F}(\Omega)+1\right|^{2} S_{x}^{\text {tech }}(\Omega)\right. \\
& \left.+\left|R_{x x}(\Omega)\right|^{2} S_{F}^{\text {tech }}(\Omega)\right)
\end{aligned}
$$

is the sum technical noise spectral density, and $S_{F}^{\text {tech }}(\Omega)$ and $S_{x}^{\text {tech }}(\Omega)$ are spectral densities of nonquantum noise sources $F_{\text {tech }}(\Omega)$ and $x_{\text {tech }}(\Omega)$. Optical losses influence, as shown in [15], can be accounted for by introducing effective quantum efficiency $\eta$ of the readout photodetector, which appears in Eq. (13).

\section{BINARY SOURCES}

The most popular and easy to implement criteria used to determine the optimal regime of GW detectors relates to the evaluation of the detection range for inspiraling binary systems of compact objects such as neutron stars and/or black holes. This method is based on the estimation of the SNR using a well-known analytical expression for the spectral density of GWs emitted by the system of two gravitationally binded inspiraling astrophysical objects (see Sec. 3.1.3 of [21]):

$$
|h(f)|^{2}=\frac{G^{5 / 3}}{c^{3}} \frac{\pi}{12} \frac{\mathcal{M}^{5 / 3}}{r^{2}} \frac{\Theta\left(f_{\max }-f\right)}{(\pi f)^{7 / 3}},
$$

where $\mathcal{M} \equiv \mu^{3 / 5} M^{2 / 5}$ is the so-called "chirp mass" of the binary system constructed from reduced mass $\mu=$ $M_{1} M_{2} / M$ and total mass $M=M_{1}+M_{2}$ of the binary system with component masses $M_{1}$ and $M_{2}$ correspondingly. One can readily see the indicative frequency depen- dence $|h(f)|^{2} \propto f^{-7 / 3}$ and inverse dependence on the distance to the system squared $r^{2}$. The upper cutoff frequency $f_{\max }$ corresponds to the period of binary system rotation on the innermost stable circular orbit when the system goes from a quasistationary rotation phase to a nonstationary merger phase. This frequency can be estimated as

$$
f_{\max } \simeq 4400 \times\left(M_{\odot} / M\right) \mathrm{Hz} .
$$

Given the GW signal shape (17) and the noise spectral density (11), it is possible to write down the optimal SNR $\rho$ which can be obtained on a given detector. As demonstrated by Flanagan and Hughes [22], the SNR averaged over all mutual orientations between the detector and the source and over both polarizations of GWs is equal to

$$
\begin{aligned}
\rho_{\mathrm{NS}}^{2} & =\frac{4}{5} \int_{f_{\min }}^{f_{\max }} \frac{|h(f)|^{2}}{S^{h}(2 \pi f)} d f \\
& =\frac{2}{15} \frac{G^{5 / 3}}{\pi^{4 / 3} c^{3}} \frac{\mathcal{M}^{5 / 3}}{r^{2}} \int_{f_{\min }}^{f_{\max }} \frac{d f}{f^{7 / 3} S^{h}(2 \pi f)},
\end{aligned}
$$

where $f_{\min }$ is the lower cutoff frequency at which binary system motion cannot be considered as stationary. In our calculations we will take $f_{\min } \simeq 10 \mathrm{~Hz}$.

In order to estimate detection range $r$ one should set a threshold SNR $\rho_{0}$ which defines the level of confidence in the detection of GWs from the binary system. Then the detection range can be written as

$$
r=\left(\frac{2}{15} \frac{G^{5 / 3}}{\pi^{4 / 3} c^{3}} \frac{\mathcal{M}^{5 / 3}}{\rho_{0}^{2}} \int_{f_{\min }}^{f_{\max }} \frac{d f}{f^{7 / 3} S^{h}(f)}\right)^{1 / 2} .
$$

Of course, all formulas we use here are obtained in the lowest post-Newtonian order of general relativity [23] that definitely limits their application area to stellar mass systems and asymptotically flat space-time background. However, for our purposes it is enough, and the most significant feature for us of the above expressions is their relative simplicity.

We calculated the detection range for the standard $\left(M=2.8 M_{\odot}\right)$ neutron stars binary system numerically considering it as a function of three parameters: $\Gamma \in$ $[500,12500] \mathrm{s}^{-1}, \quad \beta \in[-\pi / 2, \pi / 2], \quad$ and $\quad \phi \in$ $[-\pi / 2, \pi / 2]$. The number of values for each of the parameters was 193 , giving in total $193^{3} \approx 7.2 \times 10^{6}$ optical configurations. Distribution of points in parameter space was taken uniform over angle variables $\beta$ and $\phi$, and logarithmic over variable $\Gamma$. For other parameters, we used the values planned for Advanced LIGO; see Table I. To account for technical noises, we used the noise budget also planned for Advanced LIGO and generated by BENCH software [24]; see Fig. 5.

For each pair of $\Gamma, \beta$ we have maximized SNR with respect to $\phi$, thus obtaining a function of only two parameters $\Gamma, \beta$. The result of this calculation is presented in Fig. 6 as contour plots of normalized $\operatorname{SNR} \rho_{\mathrm{NS}} / \rho_{\mathrm{NS}}^{\max }$. 


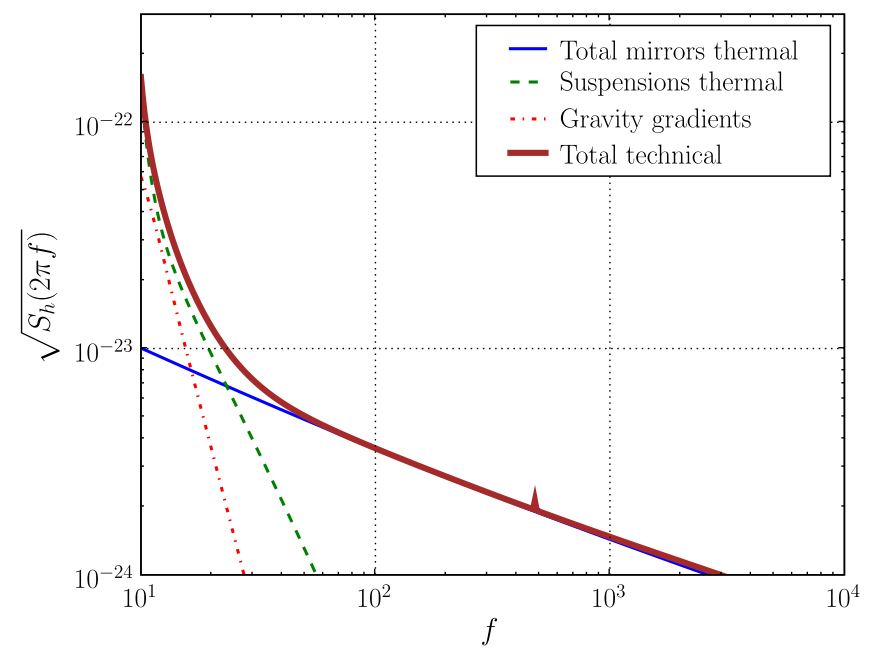

FIG. 5 (color online). Main classical noises planned for the Advanced LIGO interferometer.

Contours act in this plot as margins for regions in parameter space where SNR is higher than the certain percentage of maximal SNR $\rho_{\mathrm{NS}}^{\max }$, being indicated in the plot by the point marked as MAX. The parameter values for this maximal sensitivity point are listed in Table II.

It is easy to note flat behavior of SNR within a spacious range of parameters $\Gamma, \beta$. It arises due to two reasons. The first one is technical noises. It can be shown that in the absence of them, it is possible, in principle, to obtain arbitrary high values of SNR using a deep and narrow well in quantum noise spectral density created by means of the second-order-pole regime of the optical rigidity $[10,15]$, which corresponds to $\delta=(J / 4)^{1 / 3}$ and $\gamma \rightarrow 0$. Technical noise which has flat and smooth spectral dependence makes such excesses in the quantum noise useless. Moreover, they increase the quantum noise at other frequencies and thus decrease the sensitivity.

On the other hand, the integral character of criterion (20) allows significant variations of values of $\Gamma$ and $\beta$. The increase of $\Gamma$ decreases the quantum noise level at low

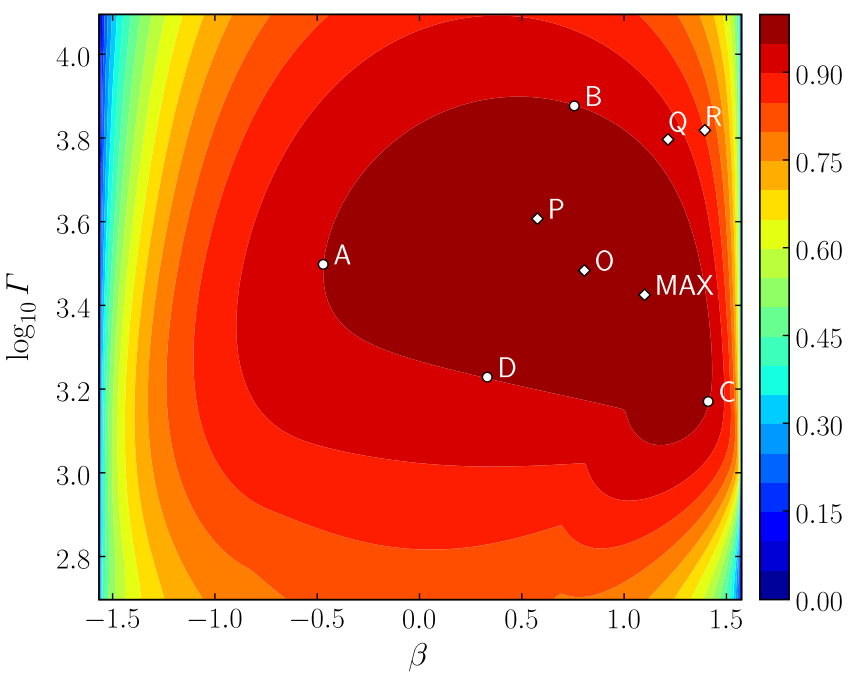

FIG. 6 (color online). Contour plot of sensitivity for standard NS-NS binaries $\rho_{\mathrm{NS}} / \rho_{\mathrm{NS}}^{\max }$ as a function of $\Gamma, \beta$. Point MAX corresponds to the sensitivity maximum, points $\mathrm{A}-\mathrm{D}$ to typical suboptimal tunings shown in Fig. 7, point O to double (NS + bursts) optimal regime, and points $\mathrm{P}, \mathrm{Q}$, and R to triple (NS + bursts + pulsar) suboptimal regimes.

frequencies $(f \lesssim 100 \mathrm{~Hz})$, but increases it at medium frequencies $(100 \lesssim f \lesssim 1000 \mathrm{~Hz})$, and vice versa. Moderate positive values of detuning $\delta$ (and hence positive values of $\beta$ ) create resonance "wells" in the noise spectral density, but increase the low-frequency noise. As a result, the integral (20) does not change significantly.

This consideration can be illustrated by Fig. 7, where quantum noise spectral densities for the optimal parameters set (point MAX in Fig. 6) as well as for four typical suboptimal ones (points A-D) are plotted.

\section{GRAVITATIONAL WAVE BURSTS}

The next type of possible GW sources are supernovae explosions and stellar core collapses, compact binary sys-

TABLE II. Optimal (MAX), double-optimal (O), and triple-suboptimal (P,Q,R) parameter values for standard NS binary, burst, and typical periodic (pulsar J0034-0534) sources.

\begin{tabular}{lcccccc}
\hline \hline & NS & Bursts & \multicolumn{4}{c}{ NS + Bursts + Pulsar } \\
& MAX & MAX & $\mathrm{O}$ & $\mathrm{P}$ & $\mathrm{Q}$ & $\mathrm{R}$ \\
\hline$\Gamma$ & 2700 & 2900 & 3100 & 4600 & 6400 & 6600 \\
$\beta$ & 1.10 & 0.57 & 0.80 & 0.52 & 1.19 & 1.39 \\
$\phi$ & -1.00 & -0.23 & -0.44 & -0.23 & -0.85 & -1.10 \\
$\rho^{2}$ & 0.84 & 0.74 & 0.79 & 0.80 & 0.94 & 0.97 \\
$\phi_{\mathrm{SRC}}$ & 1.48 & 1.52 & 1.51 & 1.54 & 1.53 & 1.53 \\
$\gamma=\Gamma \cos \beta$ & 1200 & 2400 & 2200 & 4000 & 2400 & 1200 \\
$\delta=\Gamma \sin \beta$ & 2400 & 1600 & 2200 & 2300 & 5900 & 6500 \\
$\rho_{\mathrm{NS}} / \rho_{\mathrm{NS}}^{\max }$ & 1.0 & 0.988 & 0.995 & 0.989 & 0.91 & 0.84 \\
$\rho_{\text {burst }} / \rho_{\text {burst }}^{\max }$ & 0.979 & 1.0 & 0.995 & 0.989 & 0.84 & 0.75 \\
$\rho_{\text {puls }} / \rho_{\text {puls }}^{\max }$ & 0.21 & 0.26 & 0.25 & 0.30 & 0.40 & 0.50 \\
\hline \hline
\end{tabular}




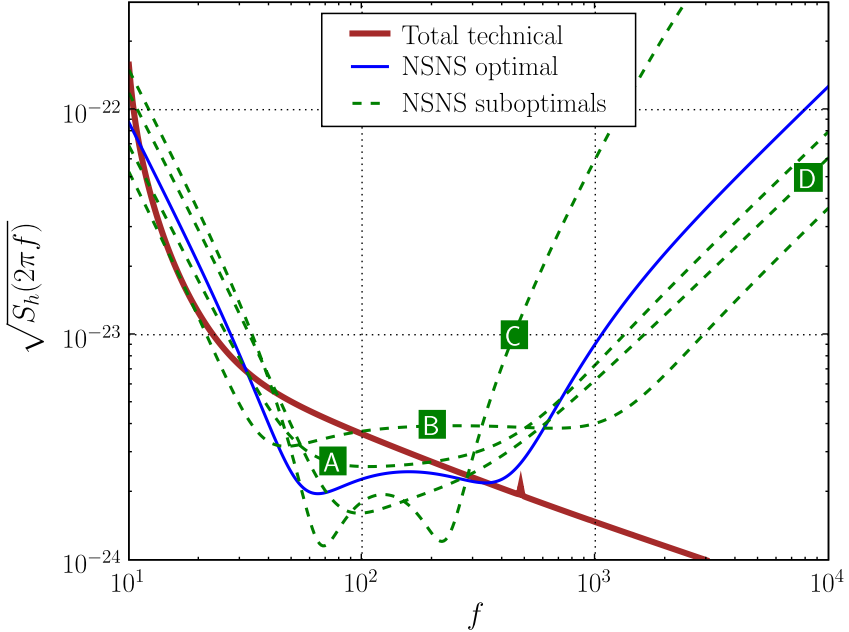

FIG. 7 (color online). Quantum noise spectral densities optimized for standard NS binary sources (point MAX in Fig. 6) and four typical suboptimal spectral densities (points A-D in Fig. 6) (see parameters in Table. III).

tem mergers [25], and other sources with not well-modeled properties which are usually called GW bursts [16-18]. For these sources, we use the simple model of a logarithmicflat signal spectrum over the range of frequencies from $f_{l}$ to $f_{h}$. By logarithmic-flat spectrum we mean that the spectrum of the GW signal $h(f)$ is proportional to $f^{-1 / 2}$ that corresponds to a constant numerator in the expression for SNR if integration is performed with respect to $\log f$ :

$$
\rho_{\text {burst }}^{2} \propto \int_{f_{l}}^{f_{h}} \frac{|h(f)|^{2} d f}{S^{h}(2 \pi f)} \propto \int_{\log f_{l}}^{\log f_{h}} \frac{d \log f}{S^{h}(2 \pi f)} .
$$

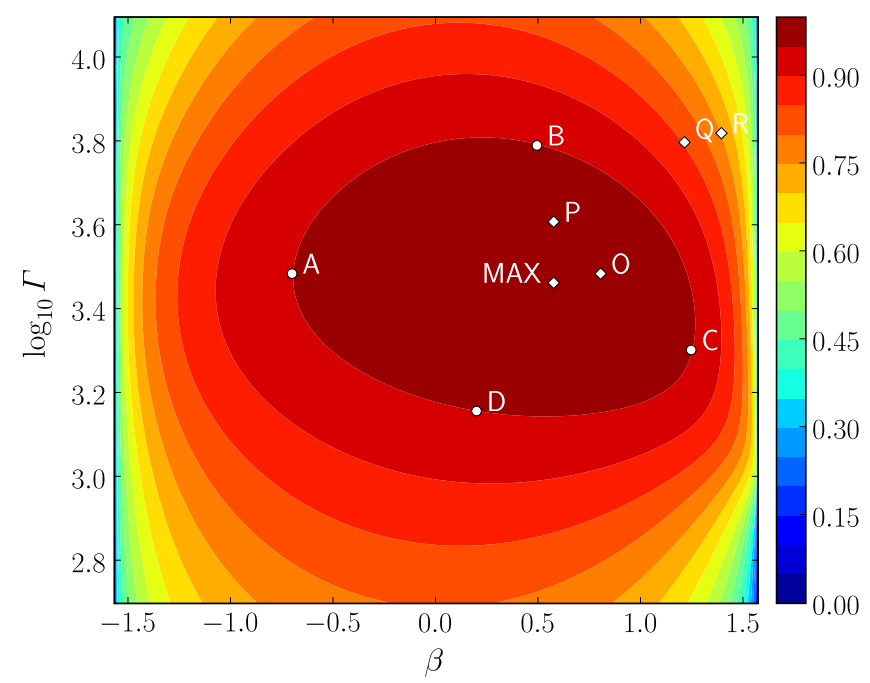

FIG. 8 (color online). Contour plot of burst sensitivity $\rho_{\text {burst }} / \rho_{\text {burst }}^{\max }$ as a function of $\Gamma, \beta$. Point MAX corresponds to the sensitivity maximum, points A-D (see parameters in Table. III) to typical suboptimal tunings shown in Fig. 9, point O to double (NS + bursts) optimal regime, and points $\mathrm{P}, \mathrm{Q}$, and $\mathrm{R}$ to triple (NS + bursts + pulsar) suboptimal regimes.

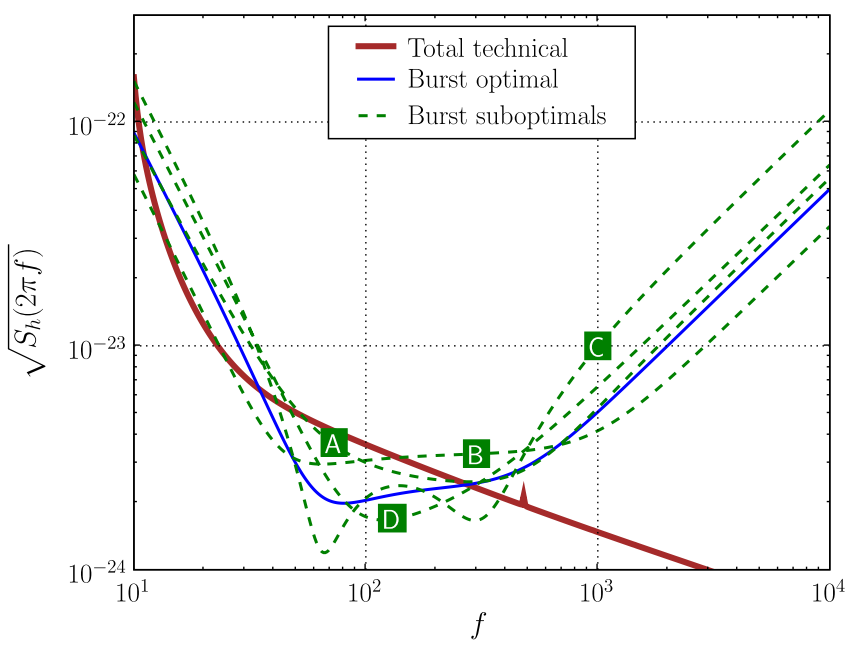

FIG. 9 (color online). Quantum noise spectral densities optimized for burst sources (point MAX in Fig. 8) and four typical suboptimal spectral densities (points A-D in Fig. 8 with parameters given in Table. III).

This way of defining SNR for burst events seems reasonable to characterize astrophysical signals with an unknown spectrum structure so that contributions from frequency (time) ranges of different order are equal (for example, contributions from $10-100 \mathrm{~Hz}$ and $100-1000 \mathrm{~Hz}$ should be the same).

In Fig. 8, function $\rho_{\text {burst }}(\Gamma, \beta)$, calculated using the same algorithm as in the previous (NS) case, and normalized by its maximal value $\rho_{\text {burst }}^{\max }$, is shown. Similar to the previous case, in Fig. 9 quantum noise spectral densities for the optimal parameters set (point MAX in Fig. 8 and for four suboptimal ones (points A-D) are plotted, and parameters values for the point MAX are listed in Table II.

It follows from Fig. 8 that function $\rho_{\text {burst }}(\Gamma, \beta)$ also has flat behavior within almost the same range of $\Gamma, \beta$ as $\rho_{\mathrm{NS}}(\Gamma, \beta)$. The main difference between the NS and burst cases, invisible in Figs. 6 and 8, stems from the existence of cutoff frequency (18) and from more steep frequency dependence of the NS signal. As a result, the NS optimization procedure leads to smaller values of angle $\phi+\beta$ that reduces quantum noise at low frequencies, while the optimization with respect to $\mathrm{GW}$ bursts requires smaller values of $\phi$ that reduces quantum noise at high frequencies

TABLE III. Optical parameters for the SRI having quantum noise plotted in Figs. 6 and 9.

\begin{tabular}{lcrcccrrr}
\hline \hline & \multicolumn{4}{c}{ NS } & \multicolumn{5}{c}{ Bursts } \\
& $\mathrm{A}$ & \multicolumn{1}{c}{$\mathrm{B}$} & $\mathrm{C}$ & $\mathrm{D}$ & $\mathrm{A}$ & \multicolumn{1}{c}{$\mathrm{B}$} & $\mathrm{C}$ & $\mathrm{D}$ \\
\hline$\Gamma$ & 3161 & 7559 & 1487 & 1700 & 3057 & 6182 & 2010 & 1438 \\
$\beta$ & -0.47 & 0.75 & 1.41 & 0.33 & -0.70 & 0.49 & 1.24 & 0.20 \\
$\phi$ & 0.97 & -0.49 & 1.49 & 0.18 & 0.47 & -0.29 & -0.57 & 0.03 \\
$\rho^{2}$ & 0.85 & 0.97 & 0.73 & 0.75 & 0.84 & 0.95 & 0.77 & 0.73 \\
$\phi_{\text {SRC }}$ & 1.55 & 1.54 & 0.09 & 0.06 & 1.55 & 1.54 & 0.03 & 0.10 \\
\hline \hline
\end{tabular}




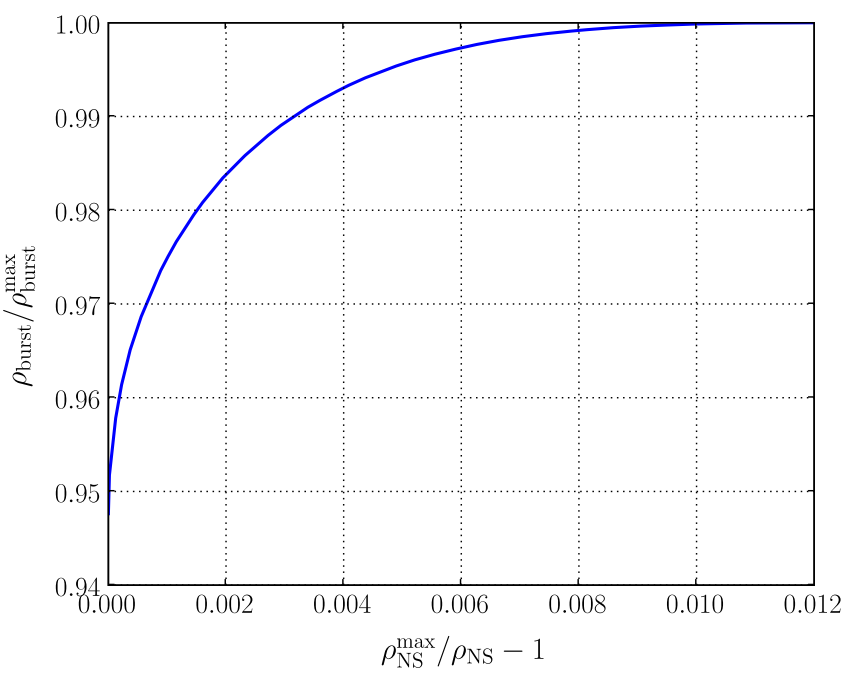

FIG. 10 (color online). Parametric plot of $\rho_{\text {burst }}(\lambda) / \rho_{\text {burst }}^{\max }$ against $\rho_{\mathrm{NS}}(\lambda) / \rho_{\mathrm{NS}}^{\max }$.

(compare Figs. 7 and 9 and the corresponding columns in Table II).

However, this difference is quite subtle, and it is evident that regimes have to exist which provide good sensitivity for both these types of GW sources simultaneously. In order to find them, we calculate values of $\Gamma, \beta$, and $\phi$, which maximize the combined normalized sensitivity

$$
G_{\mathrm{NS}+\text { burst }}(\lambda)=\lambda\left(\frac{\rho_{\mathrm{NS}}}{\rho_{\mathrm{NS}}^{\max }}\right)^{2}+(1-\lambda)\left(\frac{\rho_{\text {burst }}}{\rho_{\text {burst }}^{\max }}\right)^{2},
$$

where $\lambda$ is a Lagrange multiplier which varies in the range $[0,1]$.

The result is shown in Fig. 10, where a parametric plot of $\rho_{\text {burst }}(\lambda) / \rho_{\text {burst }}^{\max }$ against $\rho_{\mathrm{NS}}(\lambda) / \rho_{\mathrm{NS}}^{\max }$ is presented. The leftmost point on this plot corresponds to $\lambda=1$, and the

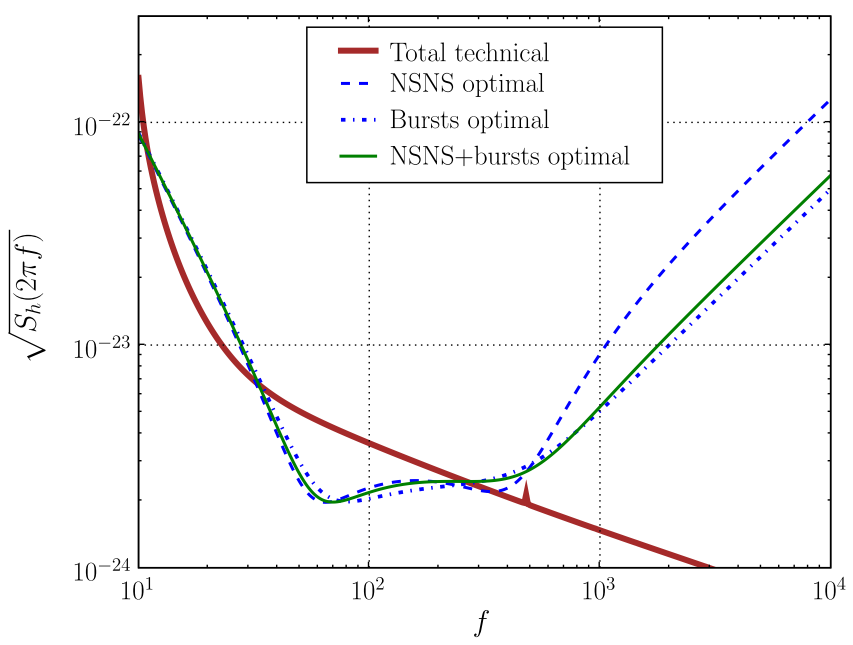

FIG. 11 (color online). Quantum noise spectral densities optimized for NS sources (point MAX in Fig. 6), burst sources (point MAX in Fig. 8) and for both of them (point $\mathrm{O}$ in Figs. 6 and 8). rightmost one to $\lambda=0$. It follows from this calculation that the tuning exists where values of $\rho_{\mathrm{NS}}$ and $\rho_{\text {burst }}$ decrease both only by $\approx 0.5 \%$ compared to their maximal values:

$$
\frac{\rho_{\mathrm{NS}}}{\rho_{\mathrm{NS}}^{\max }} \approx \frac{\rho_{\mathrm{burst}}}{\rho_{\mathrm{burst}}^{\max }} \approx 0.995 .
$$

The corresponding values of parameters $\Gamma, \beta$, and $\phi$ are listed in column $\mathrm{O}$ of Table II. This point on the plane $\{\Gamma, \beta\}$, marked as $O$, is shown also in Figs. 6 and 8.

In Fig. 11 the plot of quantum noise spectral density for this regime is presented together with plots of previously calculated optimal spectral densities for NS and burst sources. Note that all three curves are virtually indistinguishable at low and medium frequencies $(f \lesssim 600 \mathrm{~Hz}$ ), and the ones for bursts and combined NS + bursts are almost the same over all frequency ranges of interest.

\section{HIGH-FREQUENCY PERIODICAL SOURCES OF GWS}

High-frequency periodical sources of GWs, namely, millisecond pulsars, can be treated as very narrow-band almost monochromatic sources with well-defined central frequency $2 f_{\text {puls }}$ [26]. For these sources, the detection range and the SNR are simply proportional to an inverted square root of the noise spectral density at given frequency $2 f_{\text {puls }}$,

$$
r_{\text {puls }} \propto \rho_{\text {puls }} \propto \frac{1}{\sqrt{S^{h}\left(4 \pi f_{\text {puls }}\right)}} .
$$

Direct optimization of quantum noise in this case gives spectral density with very narrow and deep minimum at frequency $2 f_{\text {puls }}$, which is evidently nonoptimal for the NS and burst sources considered above. Moreover, technical noise makes it useless to have very deep minima in quantum noise spectral density, limiting $\rho_{\text {puls }}$ by the value of

$$
\rho_{\text {puls }}^{\max } \propto \frac{1}{\sqrt{S_{\text {tech }}^{h}\left(4 \pi f_{\text {puls }}\right)}} .
$$

Therefore, we optimize the following "triple-purpose" function:

$$
G_{\mathrm{NS}+\text { burst }+ \text { puls }}(\lambda, \mu)=\left[\frac{1}{G_{\mathrm{NS}+\text { burst }}(\lambda)}+\mu\left(\frac{\rho_{\mathrm{puls}}^{\max }}{\rho_{\mathrm{puls}}}\right)^{2}\right]^{-1},
$$

where $0 \leq \lambda \leq 1$ and $\mu>0$ are Lagrange multipliers. We took pulsar J0034-0534 [27] as an example of millisecond pulsars, presumably emitting narrow-band high-frequency GWs. Its barycentric rotational frequency is equal to $f_{0} \approx$ $532.7 \mathrm{~Hz}$ and the frequency of emitted GWs should be then $f_{\mathrm{GW}}=2 f_{0} \approx 1065.4 \mathrm{~Hz}$. Among the high-frequency pulsars, the distance to this one is significantly smaller com- 

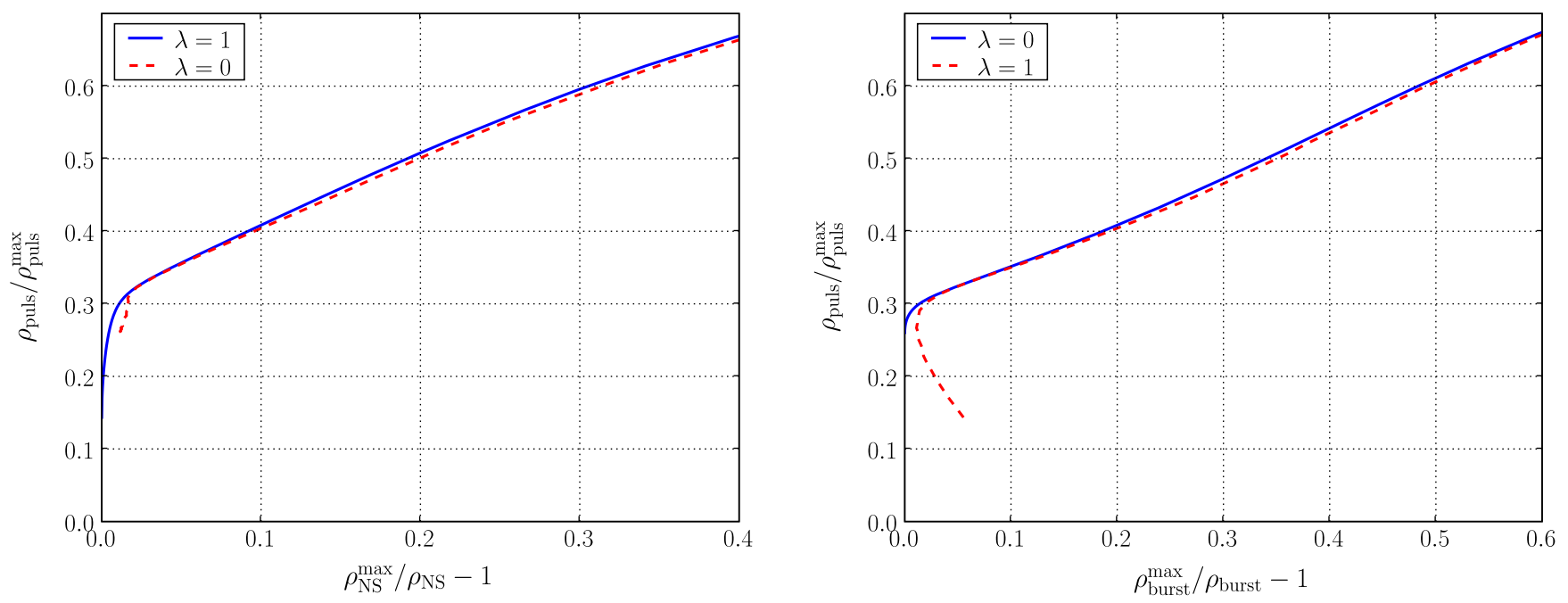

FIG. 12 (color online). Parametric plots of $\rho_{\mathrm{NS}}(\mu) / \rho_{\mathrm{NS}}^{\max }$ (left panel) and $\rho_{\text {burst }}(\mu) / \rho_{\text {burst }}^{\max }$ (right panel) against $\rho_{\text {puls }}(\lambda) / \rho_{\text {puls }}^{\max }$ for two optimization regimes: with priority to $\rho_{\mathrm{NS}}(\lambda=1)$ and to $\rho_{\text {burst }}(\lambda=0)$.

pared to other ones $(0.98 \mathrm{kpc})$ and therefore is one of the most probable candidates for GW detection.

The calculation results are presented in Fig. 10 as parametric plots of $\rho_{\mathrm{NS}}(\mu) / \rho_{\mathrm{NS}}^{\max }$ and $\rho_{\text {burst }}(\mu) / \rho_{\text {burst }}^{\max }$ against $\rho_{\text {puls }}(\mu) / \rho_{\text {puls }}^{\max }$. It follows from these plots that, despite the two-dimensional character (two Lagrange parameters $\lambda$ and $\mu$ ) of the optimization procedure, the results are virtually one dimensional, because only a very small trade-off between values of $\rho_{\mathrm{NS}}$ and $\rho_{\text {burst }}$ is possible (the lines in both planes of Fig. 12 almost coincide).

In Fig. 13 quantum noises for four characteristic regimes with optimal $G_{\mathrm{NS}+\text { burst+puls }}$ are plotted. These plots demonstrate how the optimization algorithm increases the sensitivity at the given frequency $2 f_{\text {puls }}$. The starting point $\mathrm{O}$ corresponds to the obtained parameters set in Sec. V, opti-

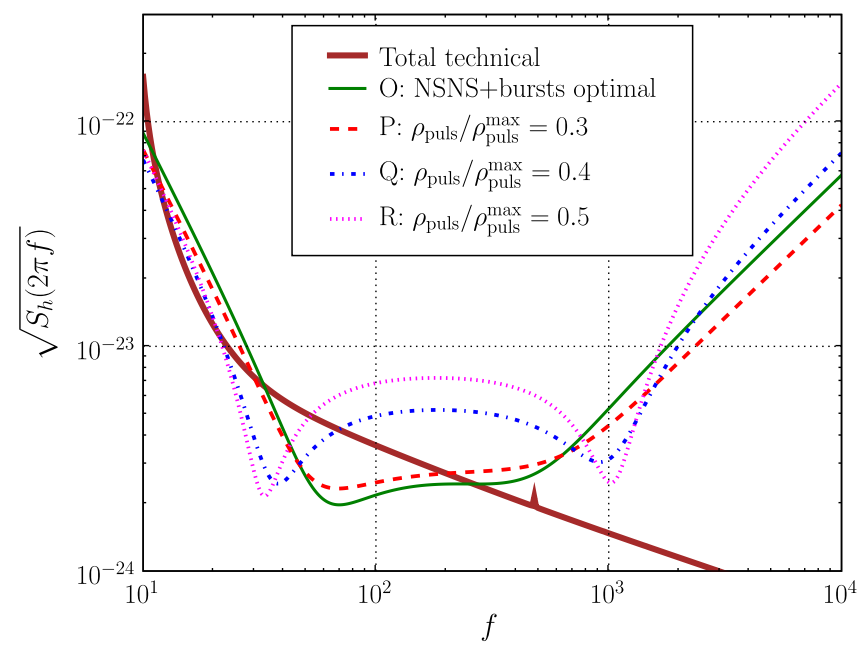

FIG. 13 (color online). Typical quantum noise spectral densities produced by the triple (NS + bursts + pulsars) optimization procedure. mized for NS and bursts signals; see Figs. 6 and 8. First, the optimization algorithm tries to suppress the quantum noise in the high-frequency range $\left(f \geqslant 10^{3} \mathrm{~Hz}\right)$ by increasing $\Gamma$ and decreasing $\beta$, see point $\mathrm{P}$ in these plots. At this stage, a noticeable gain in pulsars sensitivity $(\sim 1.5)$ can be obtained with a negligibly small $(\sim 1 \%)$ sensitivity loss for NS and burst sources (see Table II). Then, the optimization algorithm starts to "grow" local minimum at frequency $2 f_{\text {puls }}$, by increasing back $\beta$ in such a way that $\delta=$ $\Gamma \sin \beta \rightarrow 4 \pi f_{\text {puls }}$, see points Q and R. At this stage $\rho_{\mathrm{NS}}$ and $\rho_{\text {burst }}$ start to decrease noticeably (by tens of percent). However, $\rho_{\text {puls }}$ increases several times at this stage.

\section{CONCLUSION}

The results of this paper rely heavily on the estimates of the technical noise predicted for Advanced LIGO. These estimates almost definitely will be subject to change during the next few years, however, it is improbable that technical noise estimates will change significantly. Therefore, all specific values obtained here should not be considered as final ones.

The main result of this paper is not these values, but the conclusion that regimes of the signal-recycled interferometer exist which can provide good sensitivity for both binary inspiraling and burst gravitational wave sources. Moreover, triple-purpose regimes are also possible, which provide significant sensitivity gain for high-frequency periodical sources (millisecond pulsar) with only minor sensitivity degradation for binary inspiraling and burst GW sources.

Calculations presented in this paper show that in order to obtain good sensitivity for binary, burst, and, to some extent, high-frequency periodic sources, it is necessary to use large values of interferometer bandwidth $\gamma \sim(2-4) \times$ $10^{3} \mathrm{~s}^{-1} \gg 2 \times \pi 100 \mathrm{~s}^{-1}$ with significant positive detun- 
ings $\delta \sim 2 \times 10^{3} \mathrm{~s}^{-1}$; see Table II. These tunings give a smooth broadband shape of quantum noise curves, dictated by the technical noises, especially by the mirrors thermal noise which has very flat frequency dependence in the GW signals spectral range.

\section{ACKNOWLEDGMENTS}

The work of F. K. was supported in part by the NSF and Caltech Grant No. PHY-0353775. The work of I. K. and D. S. was supported by the Russian Government Grant No. NSh-5178.2006.2. The work of S.D. was supported by the Alexander von Humboldt Foundation. The authors are grateful to Yanbei Chen for extremely useful sugges- tions and invaluable counseling, to Linqing Wen, Haixing Miao, Helge Müller-Ebhardt, Henning Rehbein, and Kentaro Somiya for fruitful discussions and friendly encouragement. Special thanks to Rana Adhikari and all AdvLIGO Lab members for sharing their invaluable knowledge of specific features of real interferometers and many useful comments and suggestions that allowed us to improve this paper dramatically. The authors also would like to thank MPI für Gravitationsphysik (AEI) both in Golm and in Hannover represented by directors Professor Dr. B. Schutz and Professor Dr. K. Danzmann for outstanding hospitality and cordial reception.
[1] S. J. Waldman (LIGO Science Collaboration), Classical Quantum Gravity 23, S653 (2006).

[2] F. Acernese et al., Classical Quantum Gravity 23, S635 (2006).

[3] S. Hild (LIGO Scientific Collaboration), Classical Quantum Gravity 23, S643 (2006).

[4] M. Ando and TAMA Collaboration, Classical Quantum Gravity 22, S881 (2005).

[5] Albert Lazzarini, update from LIGO Laboratory, LIGO Document No. G070649-00-M, 2007.

[6] B. J. Meers, Phys. Rev. D 38, 2317 (1988).

[7] A. Buonanno and Y. Chen, Phys. Rev. D 65, 042001 (2002).

[8] V. B. Braginsky, A. B. Manukin, and M. Y. Tikhonov, Sov. Phys. JETP 58, 1550 (1970).

[9] V. B. Braginsky and F. Ya. Khalili, Phys. Lett. A 257, 241 (1999).

[10] F. Ya. Khalili, Phys. Lett. A 288, 251 (2001).

[11] I. A. Bilenko and A. A. Samoylenko, Moscow Univ. Phys. Bull. 3, 39 (2003).

[12] A. Buonanno and Y. Chen, Phys. Rev. D 64, 042006 (2001).

[13] A. Buonanno and Y. Chen, Phys. Rev. D 67, 062002 (2003).

[14] V. I. Lazebny and S. P. Vyatchanin, Phys. Lett. A 344, 7 (2005).
[15] F. Ya. Khalili, V. I. Lazebny, and S. P. Vyatchanin, Phys. Rev. D 73, 062002 (2006).

[16] B. Abbott et al., Phys. Rev. D 69, 102001 (2004).

[17] B. Abbott et al., Phys. Rev. D 72, 122004 (2005).

[18] B. Abbott et al. (LIGO Scientific Collaboration), Classical Quantum Gravity 24, 5343 (2007).

[19] R. Abbott et al., LIGO Technical Note LIGO-T07024701-I, 2008.

[20] V. B. Braginsky and F. Ya. Khalili, Quantum Measurement (Cambridge University Press, Cambridge, England, 1992).

[21] L. R. Yungelson and K. A. Postnov, Living Rev. Relativity 9, 6 (2006), http://www.livingreviews.org/lrr-2006-6.

[22] E. E. Flanagan and S. A. Hughes, Phys. Rev. D 57, 4535 (1998).

[23] T. Damour, B. R. Iyer, and B. S. Sathyaprakash, Phys. Rev. D 62, 084036 (2000).

[24] BENCH software, http://ilog.ligo-wa.caltech.edu:7285/ advligo/Bench/.

[25] J. G. Baker, J. Centrella, D.-I. Choi, M. Koppitz, and J. van Meter, Phys. Rev. D 73, 104002 (2006).

[26] P. Jaranowski, A. Królak, and B. F. Schutz, Phys. Rev. D 58, 063001 (1998).

[27] The Australia National Telescope Facility (ATNF) Pulsar Catalogue, http://www.atnf.csiro.au/research/pulsar/ psrcat/. 